\title{
Effectiveness of an Adventure-Based Training Programme in Promoting the Psychological Well-Being of Primary School children
}

\section{$\underline{\mathrm{Li} \mathrm{HCW}^{1}}$, Ho KY ${ }^{1}$}

${ }^{1}$ :School of Nursing, the University of Hong Kong

Background: Mental health problems in children are becoming more serious worldwide nowadays and have turned out to be a major public health concern. There has been an increase in the use of adventure-based training for school children with behavioural problems with the aim of changing their feelings, patterns of thought and behaviour. Nevertheless, empirical evidence to determine the effectiveness of such training is lacking in the existing literature, and it is uncertain whether it can promote the psychological well-being of school children.

Aim: To examine the effectiveness of an adventure-based training programme in promoting the psychological well-being of Hong Kong Chinese primary school children.

Methods: A randomized controlled trial, two-group pre-test and repeated post-tests, betweensubjects design was employed in two primary schools in a large public housing estate. A total of 120 primary grades 5 and 6 pupils were recruited in 2011, of whom 56 were randomly assigned to the experimental group participating in the adventure-based training programme and 64 to the attention placebo control group. All participants were asked to respond to the Chinese version of the Center for Epidemiologic Studies Depression Scale for Children, the State Anxiety Scale for Children, the Rosenberg's Self-Esteem Scale and the Pediatric Quality of Life Inventory.

Results: The results indicated that children in the experimental group had statistically significantly lower mean scores of depressive symptoms and anxiety and higher mean scores of self-esteem than the placebo control group. The post-hoc test confirmed that the differences in depressive symptom scores and anxiety levels between groups were statistically significant at both six and 12 months after starting the intervention. Furthermore, there was a statistically significant difference in selfesteem scores between groups at 12 months after starting the intervention.

Discussion and Conclusions: This study has addressed a gap in the literature by developing and evaluating the effectiveness of adventure-based training programme in promoting the psychological well-being of Hong Kong Chinese school children. Such programme was found to be effective, feasible in school settings and acceptable to the children concerned. Most importantly, it has been demonstrated that health care professionals should go beyond their role in collaborating with education sectors and school social workers, to promote the psychological well-being of primary school pupils in a multi-disciplinary approach. 